\title{
Non-selective optical wavelength-division multiplexing devices based on a-SiC:H multilayer heterostuctures
}

Manuela Vieira ${ }^{1,2}$, Miguel Fernandes ${ }^{1}$, Paula Louro ${ }^{1,2}$, Manuel Augusto Vieira ${ }^{1,3}$, Manuel Barata $^{1,2}$, and Alessandro Fantoni ${ }^{1}$

${ }^{1}$ Electronics Telecommunication and Computer Dept., ISEL, Rua Conselheiro Emídio Navarro, Lisbon, 1959-007, Portugal

${ }^{2}$ CTS, UNINOVA, Monte da Caparica, Caparica, 2829-516, Portugal

${ }^{3}$ Traffic Dept., CML, Lisbon, Portugal

\section{ABSTRACT}

In this paper we present results on the optimization of multilayered a-SiC:H heterostructures for wavelength-division (de) multiplexing applications. The non selective WDM device is a double heterostructure in a glass/ITO/a-SiC:H (p-i-n) /a-SiC:H(-p) /a-Si:H(-i')/a$\mathrm{SiC}: \mathrm{H}\left(-\mathrm{n}^{\prime}\right) / \mathrm{ITO}$ configuration. The single or the multiple modulated wavelength channels are passed through the device, and absorbed accordingly to its wavelength, giving rise to a time dependent wavelength electrical field modulation across it. The effect of single or multiple input signals is converted to an electrical signal to regain the information (wavelength, intensity and frequency) of the incoming photogenerated carriers. Here, the (de) multiplexing of the channels is accomplished electronically, not optically. This approach offers advantages in terms of cost since several channels share the same optical components; and the electrical components are typically less expensive than the optical ones. An electrical model gives insight into the device operation.

\section{INTRODUCTION}

Until the late 1980s, optical fiber communications was mainly confined to transmitting a single optical channel. Because fiber attenuation was involved this channel required periodic regeneration, which included detection, electronic processing, and optical retransmission. Such regeneration causes a high-speed optoelectronic bottleneck and can handle only a single wavelength. Recently plastic optical fibers (POF) [1] are drawing the attention of the industry because they are produced at very low cost and easily installed by non-specially trained people both in home networking, industrial control networks and automotive applications [2]. To improve the transmission data rate, Wavelength Division Multiplexing (WDM) can be employed. WDM enables the use of a significant portion of the available fiber bandwidth by allowing many independent signals to be transmitted simultaneously on one fiber, with each signal located at a different wavelength. Routing and detection of these signals can be accomplished independently, with the wavelength determining the communication path by acting as the signature of the origin, destination or routing. Components are therefore required to be wavelength selective, allowing for the transmission, recovery, or routing of specific wavelengths. Although they are well known for infrared telecommunication, they must be completely renewed for the transmission with the POF fibers. So, the conception of new devices for signal (de)multiplexing in the visible spectrum is a demand in this field $[3,4,5]$. This paper presents preliminary results on the applicability of multilayered a-SiC: $\mathrm{H}$ heterostructures as electrically programmable optical filters for WDM tunable devices. 


\section{EXPERIMENTAL DETAILS}

The device is a double heterostructure in a glass/ITO/a-SiC:H (p-i-n)/ a-SiC:H(-p)/a-Si:H(-i')/a-SiC:H(-n)/ITO configuration produced by Plasma Enhanced Chemical Vapor Deposition (figure1). The thickness and the absorption coefficient of the front $\mathrm{p}-\mathrm{i}-\mathrm{n}$ photodiode are optimized for blue collection and red transmittance and the thickness of the back one adjusted to achieve high collection in the red spectral range. So, both front and back diodes work as optical filters confining the blue $\left(\lambda_{B}=450 \mathrm{~nm}\right)$ and the red $\left(\lambda_{R}=650 \mathrm{~nm}\right)$ photogenerated carriers, respectively, at the front and back diodes [6].

Monochromatic beams together or one single polychromatic (mixture of different wavelength) beam impinge on the device and are absorbed, depending on their wavelength, giving rise to a time and wavelength dependent electrical field modulation across it [7].

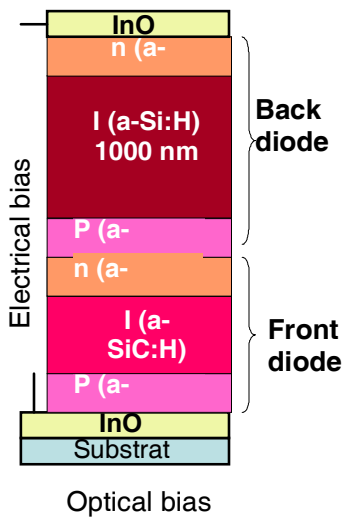

Figure 1. WDM device configuration.

The combined effect of the input signal is converted to an electrical signal, via the device, keeping the input information (wavelength, intensity and modulation frequency). By reading out, under different electrical bias conditions, the photocurrent generated by the incoming photons, the input information is electrically multiplexed or demultiplexed and can be transmitted again. In the multiplexing mode the device faces the modulated light incoming together from the fibers (monochromatic input channels) and the electronic signal is read out, under reverse bias. In the multiplexing mode a polychromatic modulated light beam is projected onto the device and the readout is performed by shifting between forward and reverse bias.

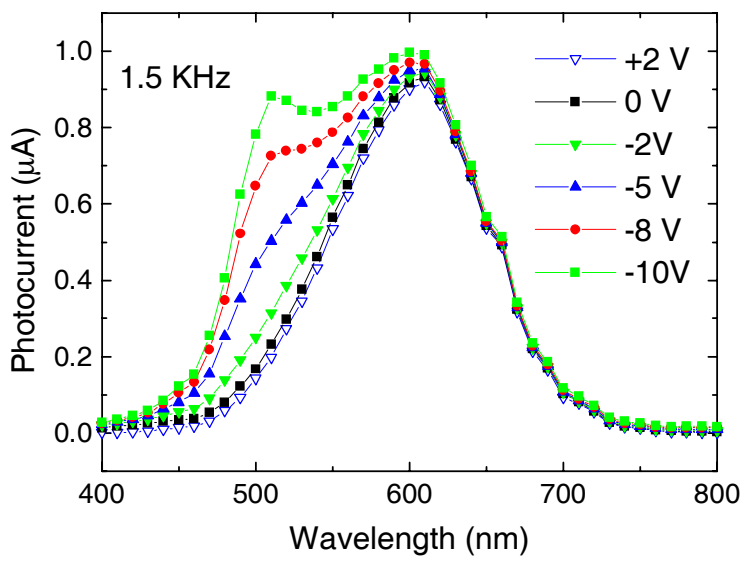

Figure 2. Spectral photocurrent under different applied voltages and modulated frequency of $1.5 \mathrm{kHz}$.

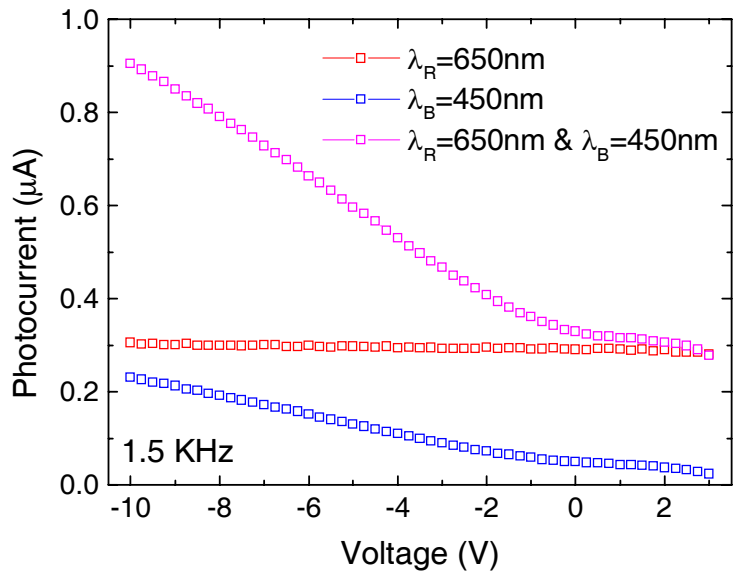

Figure 3. The ac IV characteristics under $\lambda_{R}=650 \mathrm{~nm}, \lambda_{B}=450 \mathrm{~nm}, \lambda_{R}=650 \mathrm{~nm} \&$ $\mathrm{B} \lambda_{\mathrm{B}}=450 \mathrm{~nm}$ modulated light at $1.5 \mathrm{kHz}$.

The devices were characterized through spectral response $(400-800 \mathrm{~nm})$ and photocurrentvoltage $(-10 \mathrm{~V}<\mathrm{V}<+2 \mathrm{~V})$ measurements. In figure 2 the spectral photocurrent measured at 1.5 $\mathrm{kHz}$ is displayed at different applied voltages. In figure 3 the $a c$ current-voltage characteristics under illumination are shown. In this measurement two modulated $(1.5 \mathrm{kHz})$ monochromatic 
lights; red $\left(\lambda_{R}=650 \mathrm{~nm}\right)$ and blue $\left(\lambda_{B}=450 \mathrm{~nm}\right)$ and a polychromatic one $\left(\lambda_{R}=650 \mathrm{~nm} \& \lambda_{B}=450\right.$ $\mathrm{nm})$ where impinging the device one after the other and the photocurrent was measured as a function of the voltage.

Results show that, as the applied voltage changes from forward to reverse the blue/green spectral collection is enlarged while the red one remains constant (figure 2). The collection efficiency (figure 3) under red modulated light is independent on the applied voltage while under blue or combined red and blue irradiation, it quickly increases under reverse bias. It is interesting to notice that, under forward bias, the red and the red \& blue signals are almost the same. This behavior illustrates the blindness of the device, under forward bias, to the blue component of the multiplexed signal.

\section{RESULTS AND DISCUSSION}

\section{Wavelength division (de)multiplexing}

The effect of the applied voltage $(-5 \mathrm{~V}<\mathrm{V}<+2 \mathrm{~V})$ on the output transient multiplexed signal was analyzed (figure 4). The two input wavelength channels are superimposed in the top of the figures to guide the eyes. The frequency of one was $1.5 \mathrm{kHz}$ and the other always half of this value. In figure $4 \mathrm{a}$ the signals are due to the combined effect of two beams either with the same wavelength, $R \& R\left(\lambda_{R}=650 \mathrm{~nm}\right) ; B \& B\left(\lambda_{B}=450 \mathrm{~nm}\right)$, or distinct wavelengths, $R \& B$ $\left(\lambda_{\mathrm{R}}=650 \mathrm{~nm} \& \lambda_{\mathrm{B}}=450 \mathrm{~nm}\right)$. The arrows are indicative of the external voltages: $-5 \mathrm{~V}$ (solid), $0 \mathrm{~V}$ (dash), $+2 \mathrm{~V}$ (dot). In figure $4 \mathrm{~b}$ the multiplexed $\mathrm{R} \& \mathrm{~B}$ signal is shown for electrical bias between $+2 \mathrm{~V}$ and $-5 \mathrm{~V}$.

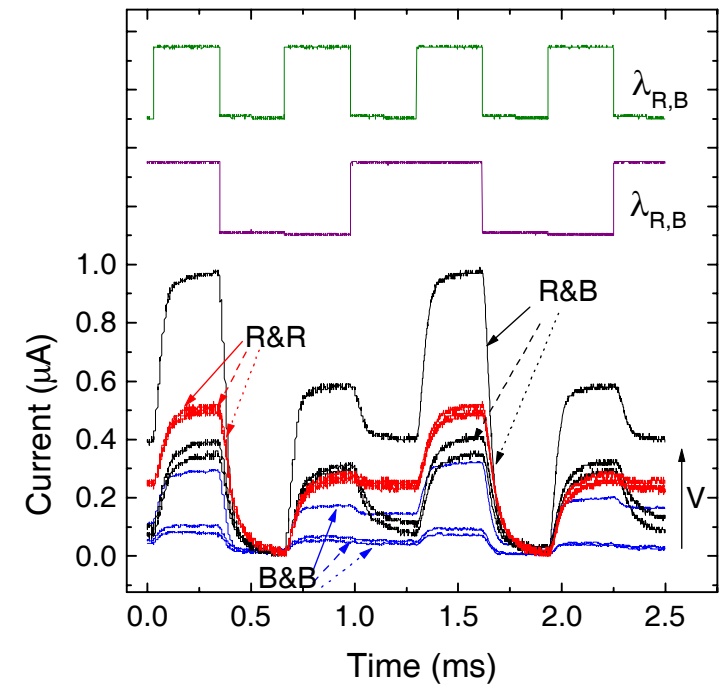

a)

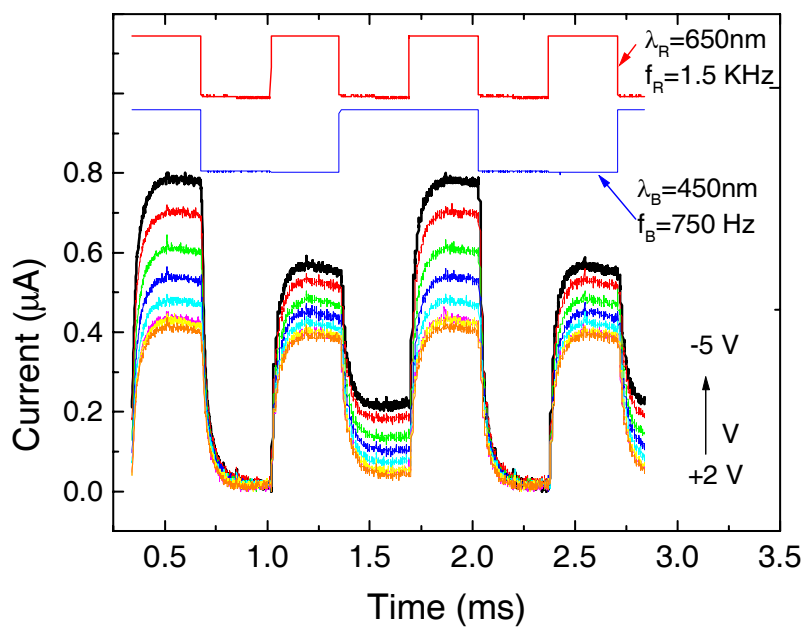

b)

Figure 4. Multiplexed signals at different applied voltages $(-5 \mathrm{~V}<\mathrm{V}<+2 \mathrm{~V})$ and input wavelengths: a) $\left.R \& R\left(\lambda_{R, R}=650,650 \mathrm{~nm}\right) ; B \& B\left(\lambda_{B, B}=450,450 \mathrm{~nm}\right), R \& B\left(\lambda_{R, B}=650,450 \mathrm{~nm}\right) . b\right)$ $\operatorname{R} \& B\left(\lambda_{R, B}=650,450 \mathrm{~nm}\right)$. The frequency of one of the input signal was $1.5 \mathrm{kHz}$ and the other was half.

Both figures show that the multiplexed signal depends on the applied voltage and on the wavelength of the input channels. If both input channels have the same wavelength (figure 4a) two opposite behaviors are observed. Under red irradiation (R\&R) the signal is high and does not depend on the applied bias, under blue irradiation $(B \& B)$ the signal increases with the negative bias and in forward bias is irrelevant. A good linearity in the signal amplitude is observed in both 
situations, showing that the joint effect of the input channels is cumulative. If both channels have different wavelength ( $R \& B$, figure $4 a$ and figure $4 b$ ), under reverse bias, there are always four levels. The highest level when both red and blue channels are ON and the lowest if both are OFF. In between, the red level (blue channel OFF) is higher than the blue level (red channel OFF). The step among them depends on the applied voltage and increases with the increase of the reverse bias. Under forward bias the blue signal goes down to zero (figure 2, figure 3 and figure 4) and the red one remains constant. The device is blind to the blue irradiation and, thus, the red signal is recovered. Results have shown that different wavelengths which are jointly transmitted must be separated to retain all the information.

The generated photocurrent was measured at two applied voltages to read out the combined spectra. The result is plotted in figure 5, in short circuit (open symbol) and under reverse bias (line). Results show that under short circuit the blue component of the combined spectra falls into the dark level, tuning the red input channel. Thus, by switching between short circuit and reverse bias the red and the blue channels were recovered.

The device acts as a charge integrator, keeping the memory of the input channel. So, it can be used as a non selective division wavelength multiplexing device, WDM.

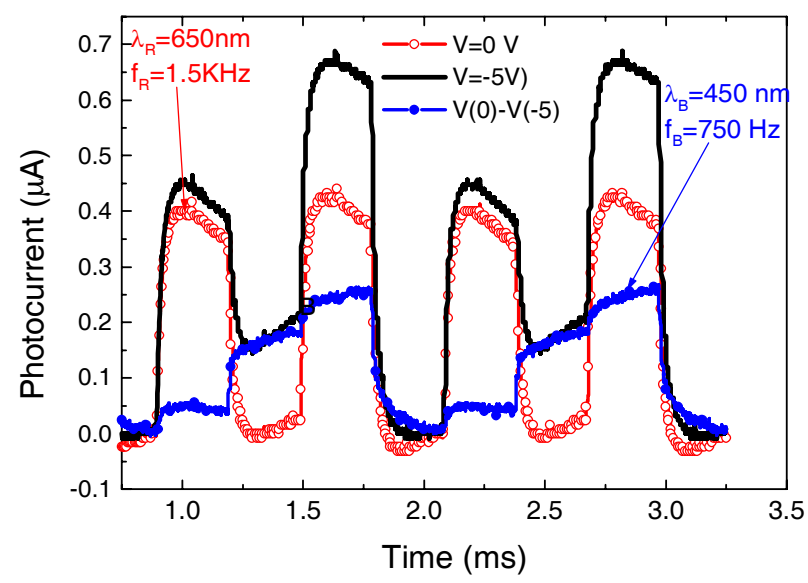

Figure 5. Blue and red wavelength division demultiplexing output channels (dot lines) for the input signal (solid line).

\section{Electrical modeling}

Based on the experimental results (figure 3, figure 4 and figure 5) and device configuration (figure 1) an electrical model was developed and supported by a SPICE simulation. Here, the internal (n-p) junction controls the current across the device. At low positive bias both front and back p-i-n junctions are forward-biased and the internal n-p reverse biased (OFF region). Under negative bias the internal junction is forward-biased ( $\mathrm{ON}$ region). The transition between both $\mathrm{ON}$ and OFF states (turning point) depends not only on the applied voltage but also on the presence of one or several wavelength input channels.

As displayed in figure 6, the device is considered to be like two phototransistors connected back to back, modeling respectively the a-SiC:H p-i-n-p and a-Si:H n-p-i-n sequences. One transistor, Q1, is pnp type and the second, Q2, npn. In order to simulate the n-p internal junction, the pnp shares its collector with the base of the npn and its base with the npn collector. Capacitors, $\mathrm{C} 1$ and $\mathrm{C} 2$, are used to simulate the capacitance transient due to the minority carrier trapped in both p-i-n junctions. A voltage source has been applied through a resistor, giving rise to a current I (R3). Two ac current sources, I1 and I2, are used to simulate the input blue and red channels photocurrents. The frequencies are the same as the ones used in the experimental work (figure 3a). In figure $6 \mathrm{~b}$ the input channels, I(I1), I(I2), the simulated multiplexed signal, I(R3) and the current across the capacitors, I(IC1), I(IC2) are shown. A good agreement between experimental and simulated results is achieved (figure 4). 

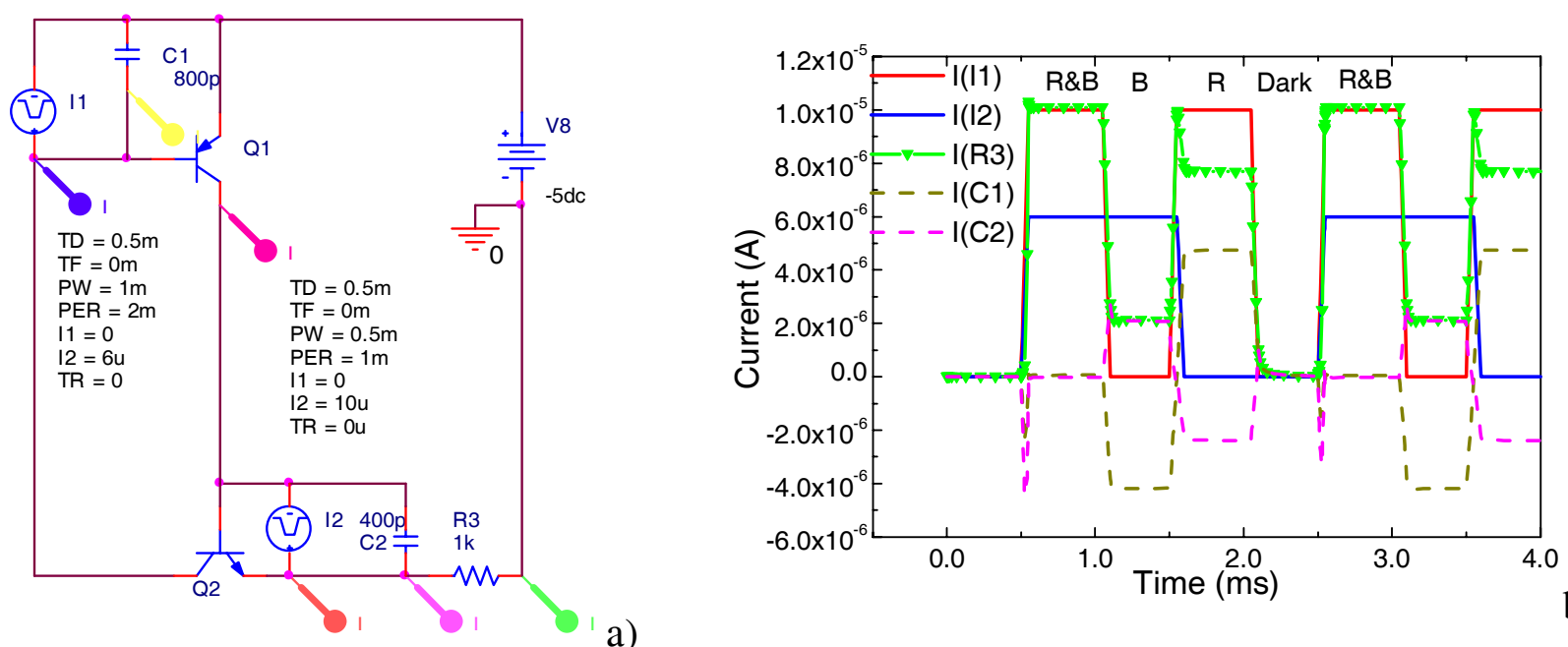

a)

b)

Figure 6. a) Equivalent electrical circuit of the pin-pin photodiode. b) Signals obtained using SPICE simulation when the red (I2) and the blue (I1) modulated lights are impinging the device.

Results show that if the device is biased negatively (-5V) Q1 and Q2 are in their reverse active regions. The $\mathrm{p}-\mathrm{n}$ internal junction is forward-biased and the external voltage drops mainly across both front and back reverse-biased junctions, mainly at the front one due to its higher resistivity. So, the external current, I, depends not only on the balance between both blue and red photocurrents (I1, I2) [8] but also on the end of each half-cycle of each modulated current. Here, the movement of charge carriers with an increase/decrease in the irradiation, results in a charging or a displacement current similar to the current $(\mathrm{i}=\mathrm{CdV} / \mathrm{dt})$ that charges the capacitors $\mathrm{C} 1$ and $\mathrm{C} 2$ in opposite ways.

In the beginning of the cycle (R\&B), I1 flows across Q1 collector towards the base of Q2 and together with the photogenerated carriers by the red light recombine or are collected (R\&B level). Under blue irradiation, I(I2)=0, only the carriers generated by the blue photons are injected into the base of $\mathrm{C} 1$ (B level). $\mathrm{C} 1$ charges positively and $\mathrm{C} 2$ negatively as a reaction to the decrease in the red irradiation. The opposite occurs under red irradiation where only the red photogenerated carriers contribute to the external current ( $\mathrm{R}$ level). Here, the capacitors recharge in an opposite way. When both red and blue lights are simultaneously OFF, the current is limited by the leakage current of both active junctions (dark level). So, once triggered, the device continues to conduct until the current through it drops below a certain threshold value, such as at the end of a half-cycle, keeping the information of the wavelength ( $R \& B, R, B, D a r k)$ and frequency $(\mathrm{f} 1, \mathrm{f} 2)$ of the impinging light. When a positive voltage is applied to turn the device from the OFF to the $\mathrm{ON}$ regions, the junction capacitance across the internal n-p junction is charged. The charging current flows through the emitter of the two transistors. The device behaves essentially as a $n p n$ phototransistor with the pnp transistor acting like a emitter-follower with a very small gain. So, under lower positive voltages the only carriers collected come from the red channel enabling the demultiplexing of the previous multiplexed signal (Fig.5).

Comparing both the experimental and the simulated results it is observed that, under negative applied voltages, the multiplexed signal keeps the memory of the single input channels. It presents four distinct levels, ascribed to the simultaneous presence of both $\left(\lambda_{1} ; \lambda_{2}\right)$; one $\left(\lambda_{1} ; 0\right)$ / $\left(0 ; \lambda_{2} ;\right)$ or none $(0 ; 0)$ optical carriers. Under positive bias the blue $\left(\lambda_{1}=450 \mathrm{~nm}\right)$ component of the 
combined spectra falls into the dark level, tuning the red $\left(\lambda_{2}=650 \mathrm{~nm}\right)$ input channel. By switching between short circuit and reverse bias the red and the blue channels were recovered.

\section{CONCLUSIONS}

Preliminary results on the applicability of multilayered double p-i-n a-SiC:H/a-Si:H heterostructures, as non selective WDM devices, were presented. Two modulated input channels were transmitted together, each one located at different wavelength and frequencies. The combined optical signal was analyzed by reading out the photocurrent generated across the device. Results show that by switching between positive and negative voltages both the input channels can be recovered.

A physical model supported by an electrical simulation gives insight into the device operation. More work has to be done in order to increase the number of different independent wavelengths that can be simultaneously transmitted and multiplexed using the multilayered non selective WDM device. Digital home appliance interfaces, home and car network and traffic control applications are foreseen due to the low cost associated to the amorphous a-SiC:H and POF technologies.

\section{ACKNOWLEDGMENTS}

This work has been financially supported by POCTI/FIS/58746/2004 and Fundação Calouste Gulbenkian. The authors tank G. Lavareda and N. de Carvalho by the device deposition.

\section{REFERENCES}

1. M. G. Kuzyk, Polymer Fiber Optics, Materials Physics and Applications, Taylor and Francis Group, LLC; (2007).

2. M. Kagami. "Optical Technologies for Car Applications Innovation of the optical waveguide device fabrication". Optical communications - perspectives on next generation technologies, October 23-25, 2007 in Tokyo, Japan.

3. S. Randel, A.M.J. Koonen, S.C.J. Lee, F. Breyer, M. Garcia Larrode, J. Yang, A. Ng'Oma, , G.J Rijckenberg, H.P.A. Boom. "Advanced modulation techniques for polymer optical fiber transmission”. proc. ECOC 07 (Th 4.1.4). (pp. 1-4). Berlin, Germany, (2007).

4. M. Haupt, C. Reinboth and U. H. P. Fischer. "Realization of an Economical Polymer Optical Fiber Demultiplexer", Photonics and Microsystems, 2006 International Students and Young Scientists Workshop, Wroclaw, (2006).

5. H.P.A.v.d.Boom, W. Li, G.D. Khoe. "CWDM Technology for Polymer Optical Fiber Networks". Proceedings Symposium IEEE/LEOS Benelux Chapter, Delft, The Netherlnads, (2000).

6. M. Vieira, A. Fantoni, M. Fernandes, P. Louro, G. Lavareda and C.N. Carvalho, Thin Solid Films, 515, Issue 19, 7566-7570, (2007).

7 M. Vieira, M. Fernandes, J. Martins, P. Louro, R. Schwarz, and M. Schubert, IEEE Sensor Journal, 1, 158-167, (2001).

8. E. S. Yang, "Microlectronic devices", Chap. 5, Department of Electrical Engineering, Colombia University, Mc Graw-Hill, Inc. (1988). 